ANL $-\mathrm{HEP}-\mathrm{CP}--88-57$

DE89 009873
ANL-HEP-CP-88-57

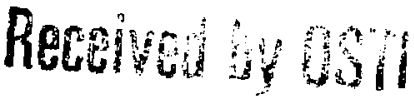

APR 141989

WAKE FIELD ACCELERATION EXPERIMENTS

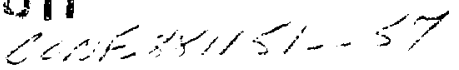

J. D. SIMPSON

High Energy Physics Difision, Argonne lational Laboratory, Argonne, IL 60439

Introduction

Where and how will wake field acceleration devices find use for other than, possibly, accelerators for high energy physics? I don't know that this can be responsibly answered at this time. What I can do is describe some recent results from an ongoing experimental program at Argonne which support the idea that wake field techniques and devices are potentially important for future accelerators. Perhaps this will spawn expanded interest and even new ideas for the use of this new tecinology.

The Argonne program, (1) and in particular the Advanced Accelerator Test Facility (AATF), has been reported in several fairly recent papers and reports. $(2-3)$ But because this is a substantially new audience for the subject, I will include a briaf review of the program and the facility before describing experiments.

Wake Fields Acceleration

What do we mean by "wake field" acceleration? Consider a bunch of charged particles moving through some structure or media. Furthermore, for simpliclty, assume it to be highly relativistic, i.e. $B=1$, and infinitesimally short. The interaction of this moving charge with its environment produces an energy change (loss) per unit length which can be expressed as

$$
U_{d}=-N_{l}^{2} e^{2} \omega(\xi=0)
$$

where $N_{1}$ is the number of particles in the bunch, and $\omega(\xi)$ is the wakefield at the position $\xi=z$ - ct relative to this bunch. A similar trailing bunch with $\mathrm{N}_{2}$ particles experiences an energy change given by

$$
U_{\omega}=-N_{1} N_{2} e^{2} \omega(\xi)-N_{2}^{2} e^{2} \omega(0)
$$

irom energy conservation it follows that the wake field at this trailing bunch due to the first bunch must satisfy

$$
|w(\xi)| \leq 2 w(0)
$$

On a "per particle" basis, this means that the ratio of accelerating gradient experienced by the trafling pulse to the decelerating gradient felt by the leading pulse is

$$
R \equiv \frac{U_{W} N_{1}}{U_{d} N_{2}} \leq 2-\frac{N_{2}}{N_{1}}
$$

This ratio, frequently referred to as the transformer ratio, is limited to two or less and is called the Wake field Theorem. At first flance, this 
result takes some of the wind out of the concept that an intense driving beam pulse can easily catapult a trailing, somewhat less intense pulse to "gee whiz" energies. There are ways to get around this limitation as we shall see. The theorem assumes that 1) the two beam pulses are colinear, or at least have the same $\omega(\xi), 2)$ the pulses have the similar longitudinal charge

distribution, and 3) the environent has linear response. Non-compliance with any of these assumptions can be used to obtain transformer ratios considerably greater than two.

Now we have a functional description of wake field acceleration; acceleration of particles directly by the residual fields, the wake fields, produced by a leading bunch travelling in a structure or mediun.

The AATF Facility

Various wake field acceleration schemes have been proposed over many years, principally as schemes to bulld, fltra-high energy Iinear colliders. They have used spejial cavity structures $(4)$ as well as plasma as the wake-forming element, $(5-6)$ and have formed the basis of a whole branch of advanced accelerator physics. About three years ago a new test facility, specifically designed to experimentally study wake fleld acceleration, was approved for constiuction at Argonne. Figure I shows a plan view of the facility which has now been in operation for about a year. An extsting $20 \mathrm{MeV}$ electron linac owned and operated by Argonne's Chenistry Division serves as the primary beam source. The facility produces pulse doublet from a single linac pulse. The leading pulse is very short ( $<20 \mathrm{ps}$ ) and intense (several $\mathrm{nC}$ ). The trailing pulse, which may be precisely positioned behind the first from zero delay out to about a nanosecond, is also short but several orders of magnitude less Intense, and is about $15 \mathrm{MeV}$.

These pulse doublets are passed through an experiment test section and into a wide spectrum spectrometer. Measurement of the energy shift of the trailing "witness" pulse as a function of bunch separation yields inforioation about the wake field produced by the leadlng "driver" pulse. Furthermore, if the two beams are adjusted to be slightly non-colinear, deflecting fields can also be measured by observing the motion of the witness in the non-bend plane of the spectrometer. Further Information on the design and performance of this facility can be found in the referenced mateilal.

\section{AATF Experiments}

Two inftial experiments were envisioned for the AATF. The first was measurement of the wake fields in simple iris loaded cavity structures. These measurements, now published, $(7)$ represented the first ever direct measurements of wake fields using a beam probe technique. Because the experiment was calculable, it also served as rerification of facility performance and calibration. Structures of this type had been proposed for wake field acceleration of electrons using high energy proton pulses as drivers, and this created added interest in the experiment.

The second experiment, also published ${ }^{(8)}$, represented an important milestone in advanced accelerator progress. For many years there had been proposals that the plasma waves produced by the passage of a beam pulse could yield large accelerating gradients. Beam-plasma interactions were well 
studied analytically and by numerical simulations, and it was most appropriate that the concept have experimental support. The results agreed well with theory and for the first tine ever demonstrated acceleration of a particle beam by plasma wake fields.

I now wish to report on more recent experimental results obtained using the AATF. These are on dielectric wake field acceleration (DWFA) and on nonlinear plasma wake field acceleration (PWFA) studies.

Figure 2 is a schematic representation a DWFA. Its construction is extremely simple, consisting only of a tube of dielectric material inside a conducting pipe. As a relativistic driver pulse travels along the axis of the structure, Cerenkor radiation in the dielectric excites allowed E-M modes. The excitation amplitude of each mode depends upon the geometry and the bunch current distribution. All modes have a phase velocity equal that of the driver beam, ${ }^{\circ} \mathrm{c}$. This boundary problem has been solved for a linear dielectric gase and the resulting predictions compared with AATF

experiments $(9)$ for three different dielectric materials. Figures 3 and 4 show such a prediction and the corresponding experimental results for a polystyrene dielectric. The remarkable agreement testifies to the capability of this measuring technique.

Typical measured accelerating gradients in these DWFA experiments, in which the driver contained about $2 \mathrm{nC}$ of charge and was 20 ps long, were about $0.5 \mathrm{MeV}$ per meter. This is quite modest by conventional linac standards, but the predicted performance were the puise $100 \mathrm{nC}$ and 10 ps is $150 \mathrm{MeV}$ per meter. Such driver beams are quite feasible and are, in fact, specified in an improvement plan for our facility which $I$ will discuss later.

As mentioned earlier, one way to exceed a transformer ratio of 2 in a DWFA may be to use a non-linear dielectric material such as a ferroelectric. We are interested in the possibility that such a dielectric can produce electromagnetic shock waves with a resulting large enhancement of the peak wake field. Some progress has been made in analytically solving this problem ard work is continuing. We may, in the meantime, carry out experiments using, for example, barium titanate. Should this idea succeed, the severe requirement of having ultra-short driver bunches will be somewhat relaxed.

Let me now turn to recent plasma wake field experiments, $(10)$ in which we already have measured the onset of non-linear effects. Host treatments of plasma wake field generation are conducted in a linearized framework in which the electron density variation, $n_{1}$, is pertubatively small relative to the equilibrium plasma electron density, $n_{0}$. Under these conditions the remant longitudinal electric field in the wake is purely sinusoidal with frequency " $p$ " phase velocity $c$, and therefore wavelength $\lambda=2 \star p i \star c / \omega_{p}$. Things become much more complicated as $a_{1}$ becomes, say, more than $25 \%$ of $\mathrm{p}_{0}$. The wake plasma electron dersity wave then contains harmonics of the fundamental in relative amplitudes which depend upon the ratio $n_{1}$ to $n_{0}$. This is a result from non- relativistic, one dimensional theory. We are not aware of any twoor three-dimensional non-linear treatments of PWFA.

If one considers the driver pulse parameters currently available at the AAT, one concludes that there is insufficient excitation for non-linear plasma behavior. The reason that non-linear response 1s, in fact, generated 
Is that the driver beam self-pinches ciue to charge neutralization within $10 \mathrm{~cm}$ or so in the plasma to a beam diameter approximately one third that of the unpinched beam. The resulting order-of-magnitude increase in charge density is sufficient.

Figure 5 shows the longttudinal wake field vs witness delay from a data scan in which the linac intensity momentarily dropped. During the period of low intensity the wake potential was nicely sinusoidal, as expected from linear theory. When the linac intensity returned to a higher level, the wake potential returas to a somewhat "sawtooth" form, a signature of non-linear plasma oscillations. Also note how the relative wake field amplitudes, high to low Intensity, are considerably larger than the driver pulse charge ratios. This is an example of fleld enhancement due to non-linear effects in the wake producing medium.

Measurement of deflecting wakes (beams slightly misaligued) show another signature of a non-linear wave: a series of parabola-like waves forms. This can be seen in Figure 6. Furthermore, Fourier transforms of the longitudinal and transverse wakes are the same, Figure 7, as expected. The accelerating gradient observed in this experiment exceeded $5 \mathrm{MeV}$ per meter and was produced by a driver bea!n of slightly less than $4 \mathrm{nC}$.

We have accumulated quite a bit of experimental data using the AATF, and will conduct several new experiments on DWFA and non-linear PWFA in upcoming months. What has become clear is that the wake field accelerating method is promising enough to warrant upsicaled demonstration.

What Next?

A feastbility study for a major improvernent to the AATF is almost complete. The heart of the improvement is utilization of emerging photocathode technology to provide driver capability greatly exceeding that now ayailable to us. Beam pulse charges of $100 \mathrm{nC}$ in $10 \mathrm{ps}$ long bunches appear quite feasible based on our study to date.

When such driver pulses are available, it is estimated that acceleration gradients in excess of $1 \mathrm{GeV}$ per meter would be produced in a plasma density of only $10^{14}$ per $\mathrm{cm}^{3}$, a density which can be obtained in our existing hollow cathode discharge plasma cell. Non-linear response in the DWFA is also more likely posstble in a greater variety of material.

In Conclusion

The use of wake field acceleration for high energy physics accelerators is a strong possibility and as stated earlier, has been the impetus to this area of accelerator research. I certainly expect there to be applications outside of that branch of science other than it being perhaps the world's most expensive way to measure dielectric constants. Perhaps we have a new tool for pure plasma physics. Maybe compact, high gradient laboratory accelerators will be possible. I hope that this short review of our work has been informative and useful. 


\section{References}

1. The responsible group at ANL includes W. Ga1, R. Konecny, S. Mtingwa, J. Norem, J. Rosenzweig, P. Schoessow, and J. Simpson.

2. Progress Report on ANL Adyanced Accelerator Test Facility, R. Konecay, J. Maclachlan, J. Norem, A. Ruggiero, P. Schoessow, and J. Simpson, ANL-HEPCP-86-46, Proceedings of the 1986 Linac Conference, SLAC, 434 (1986).

3. A Pulse Compression System for the ANL $20 \mathrm{MeV}$ Linac, G. Mavrogenes, J. Norem and J. Simpson, ANL-HEP-CP-86-45, Proceedings of the 1986 Linac Conference, SLAC, 429 (1986).

4. Mixing Staging, and Phasing for a Proton-Driven Wake Field Accelerator, W. Ga1, A. Ruggiero, J. Simpson ANL-HEP-CP-87-14, Proceedings of the 1987 Particle Accelerator Conference, Washington, DC, edited by E. Lindstrom and I.S. Taylor, 1,118 (1987).

5. Accelerator of Particles in Plasma, Y. Feiaberg, Atomic Energy, p. 431 (1959).

6. R.D. Ruth et al., Particle Accelerators 17, Nos. 2-4, 171 (1985).

7. Direct Measurement of Beam-Induced Flelds in Accelerating Structures, W. Ga1, R. Konecny, J. Norem, P. Schoessow, J. Simpson, H. Figueroa, A. Ruggiero, ANL-HEP-CP-88-04, Phys. Rey. Lett. 60, 21 (1988).

8. Experimental Observation of Piasma Wake-Field Acceleration, H. Figueroa, W. Gai, R. Konecny, J. Norem, P. Schoessow, J. Simpson, J. Rosenzweig, D. Cline, B. Cole, ANL-HEP-CP-88-08, Phys. Rev. Lett. 60, 98 (1988).

9. Experimental Demonstration at Wake-fleld Effects in Dielectric Structures, W. Gai, P. Schoessow, B. Cole, R. Konecry, J. Norem, J. Rosenzweig, J. Simpson, ANL-HEP-CP-88-36, submitted to Phys. Rev. Lett. (1988).

10. Experimental Measurements of Nomlinear Plasma Wakefields, J. Rosenzwefg, P. Schoessow, B. Cole, W. Ga1, R. Konecny, J. Norem, J. Simpson, ANL-HEPCP-88-43, submitted to Phys. Rev. Lett. (1988).

\section{DISCLAIMER}

This report was prepared as an account of work sponsored by an agency of the United States Government. Neither the United States Government nor any agency thereof, nor any of their employees, makes any warranty, express or implied, or assumes any legal liability or responsibility for the accuracy, completeness, or usefulness of any information, apparatus, product, or process disclosed, or represents that its use would not infringe privately owned rights. Reference herein to any specific commercial product, process, or service by trade name, trademark, manufacturer, or otherwise does not necessarily constitute or imply its endorsement, recommendation, or favoring by the United States Government or any agency thereof. The views and opinions of authors expressed herein do not necessarily state or reflert those of the United States Government or any agency thereof. 


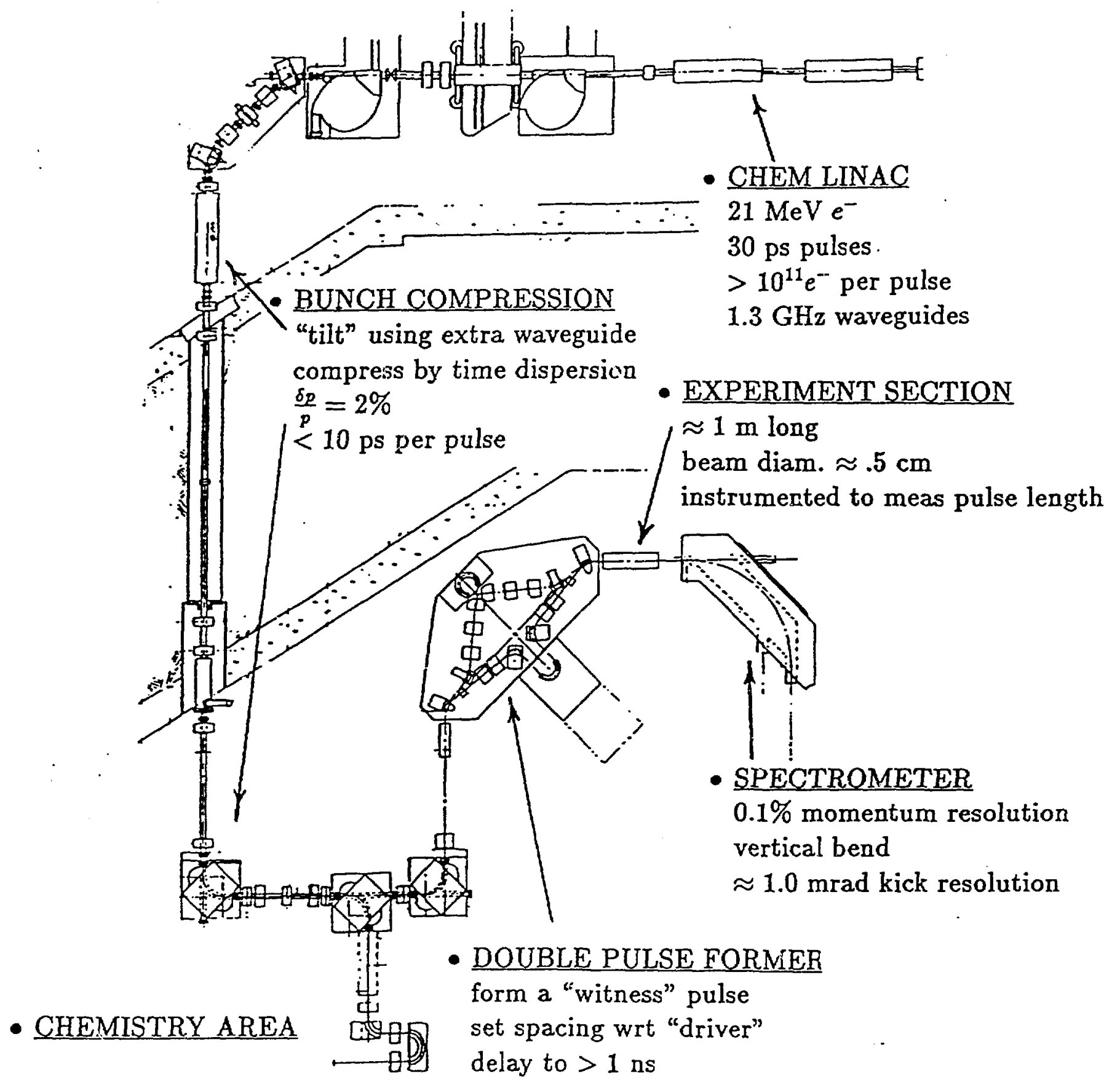

Figure 1 Plan layout of AATF showing the Linac, pulse compression system, double pulse forming/delay system, section for experiments, and high resolution spectrometer. 


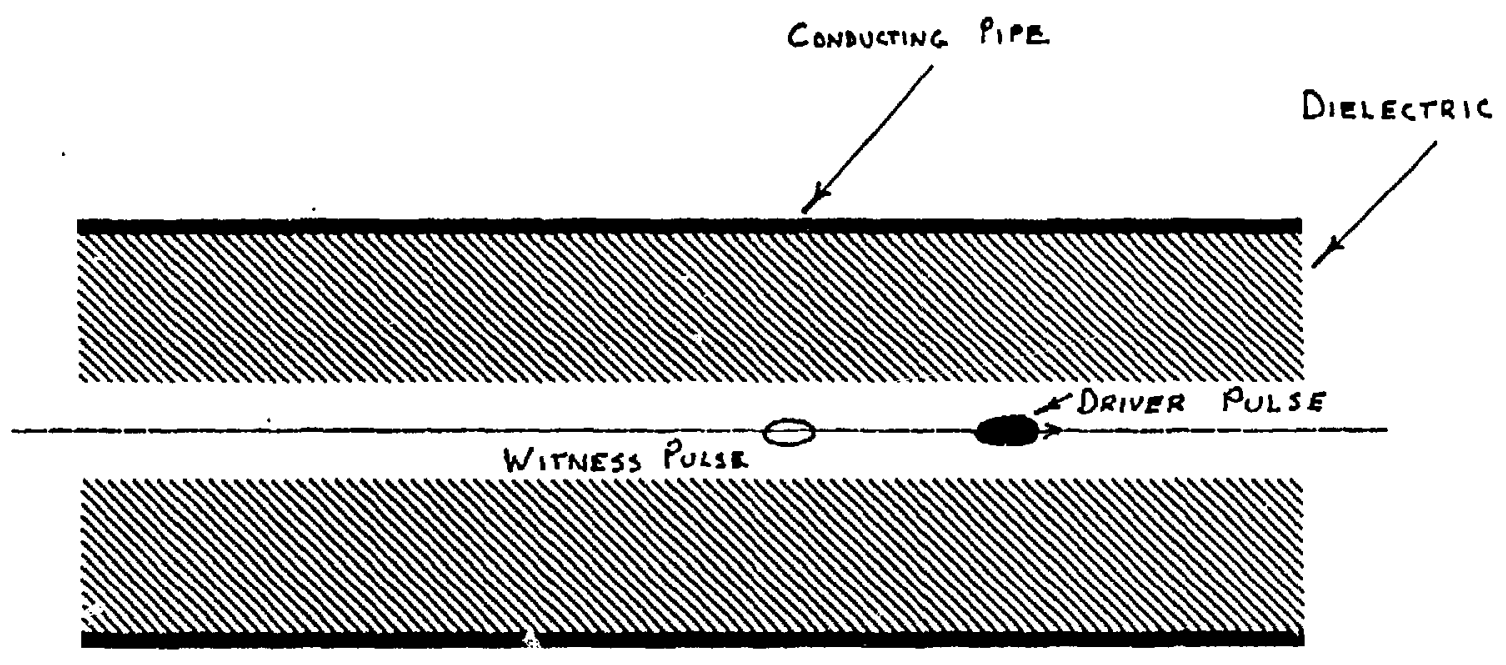

Figure 2 Schematic representation of a Dielectric Wake Field Accelerator (DWFA) section 


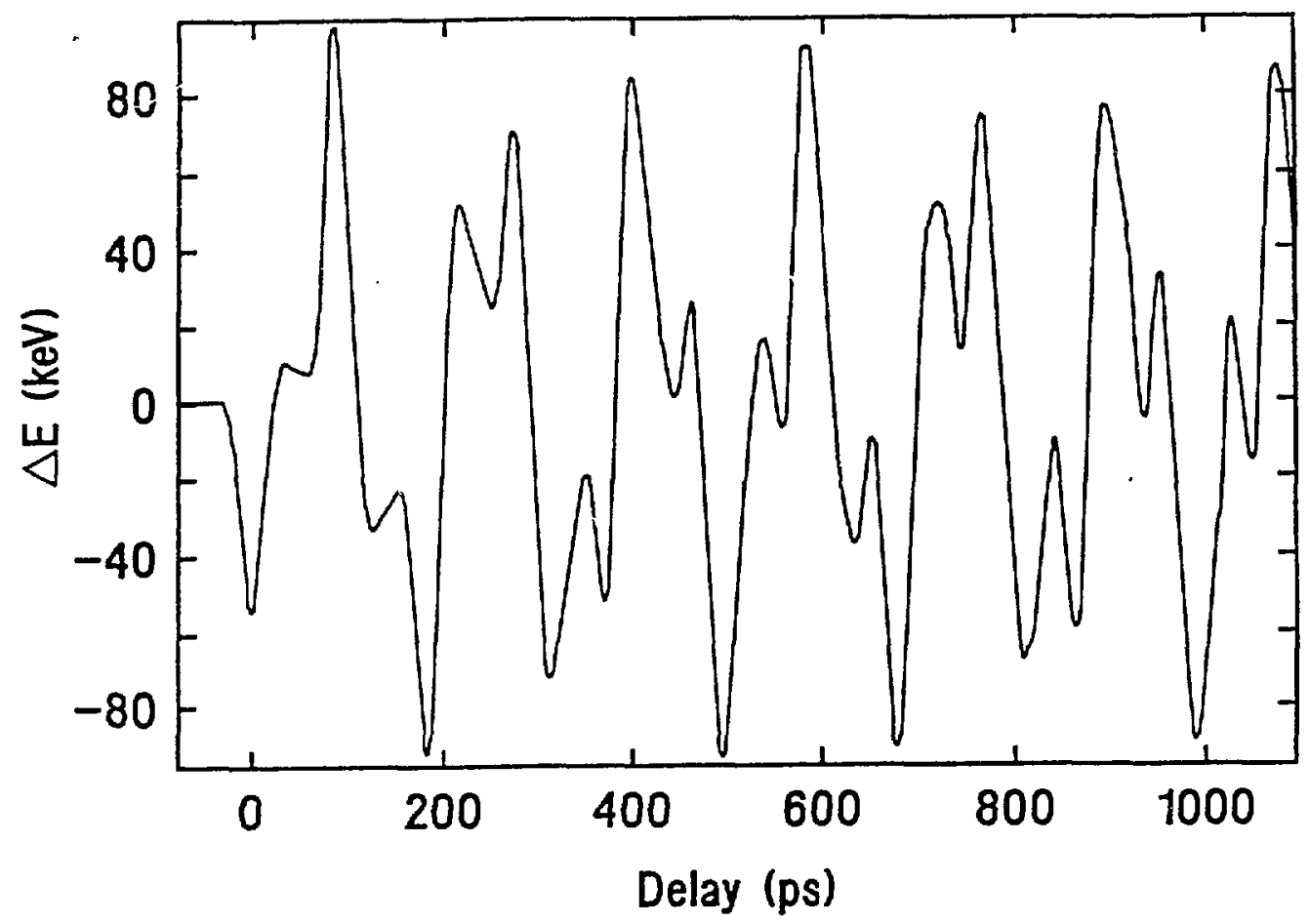

Figure 3 Calculated wake field in a DWFA using
polyethylene $(\varepsilon \simeq 5.9)$

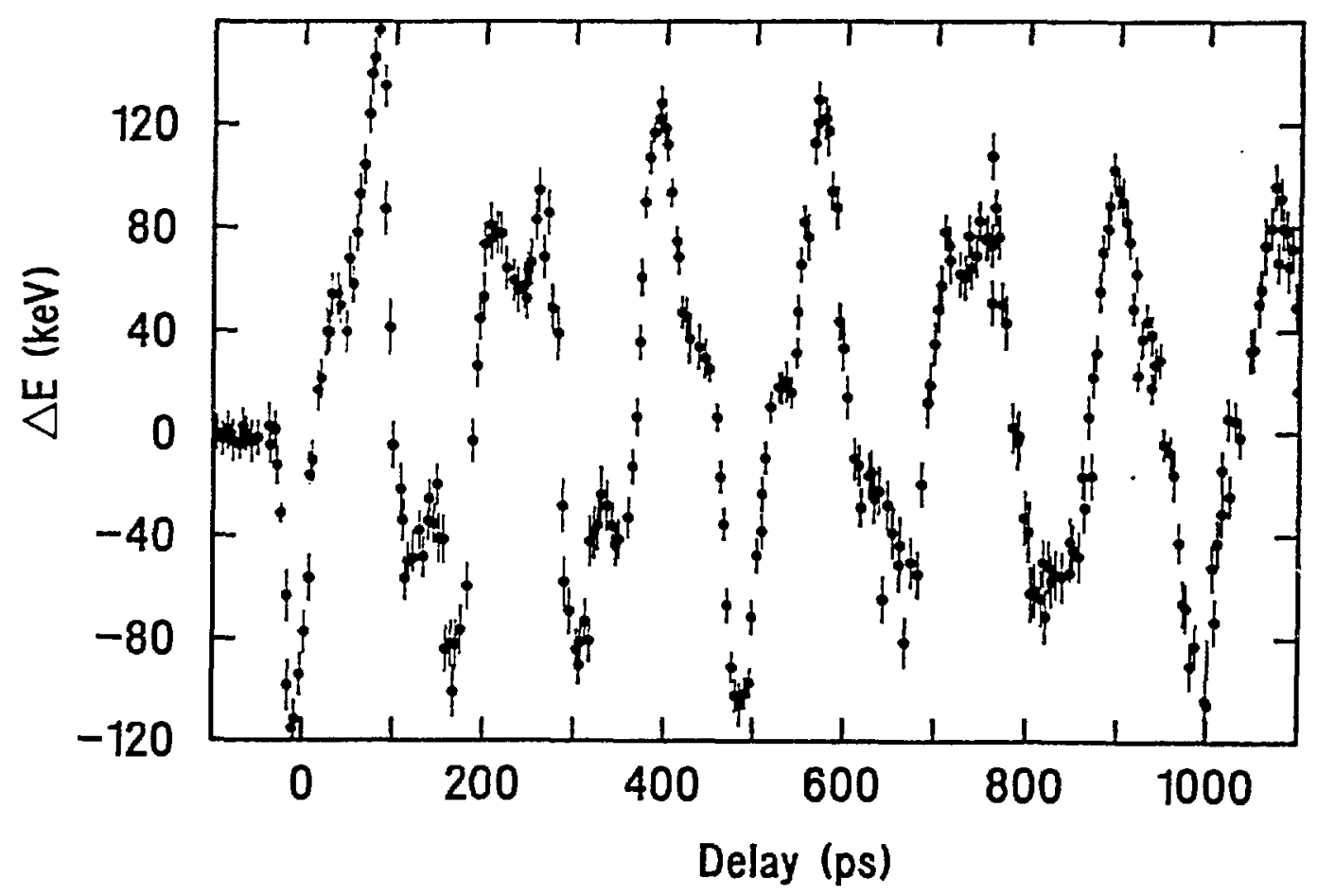

Figure 4 Measured wake field in the DWFA. Predicted high order modes (Fig. 3) are quite evident. 


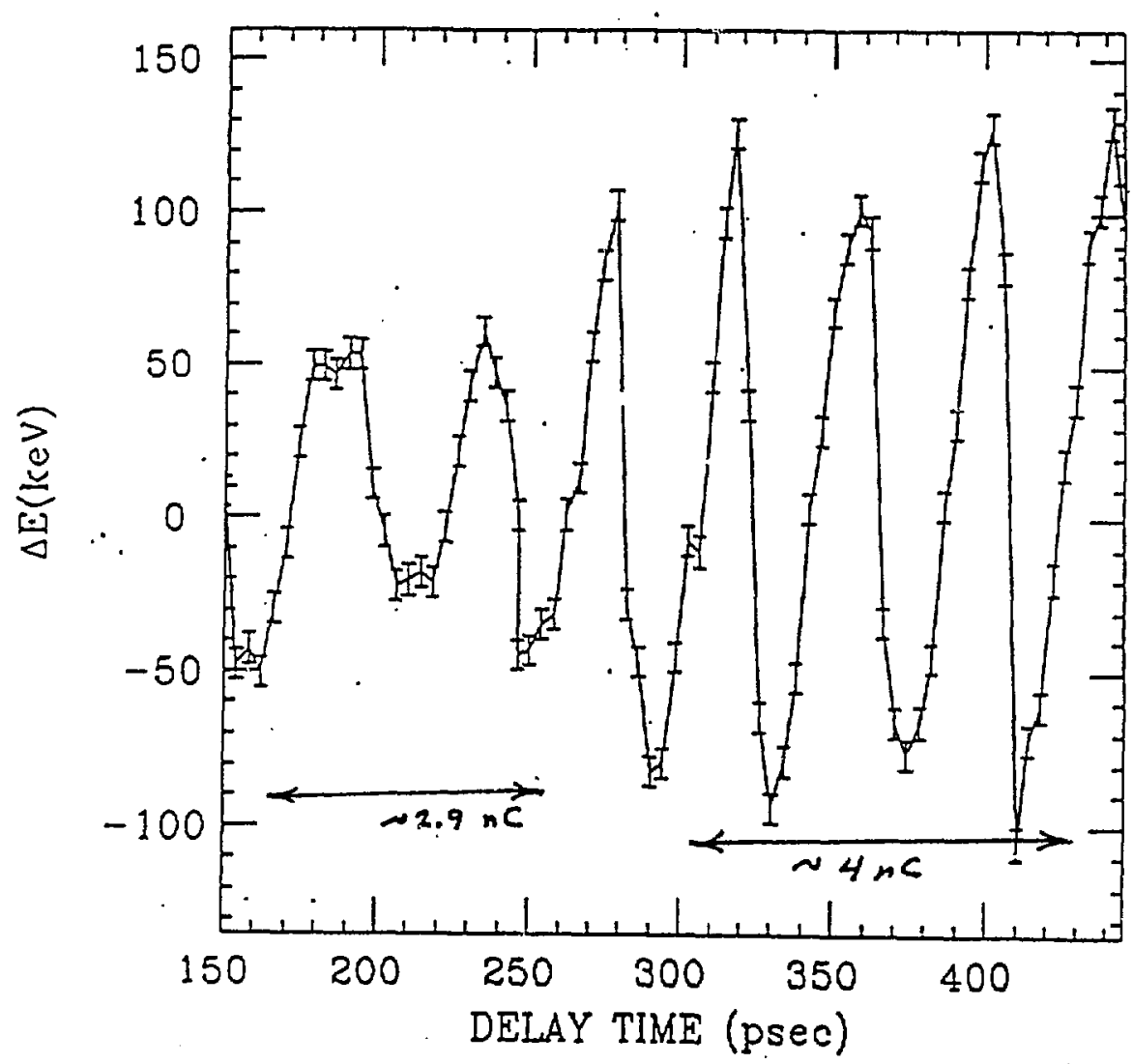

Figure 5 Plasma wake fields measured during a scan when the driver current was temporarily low. Note the disproportional increase in the field amplitudes as compared to the driver amplitudes. 


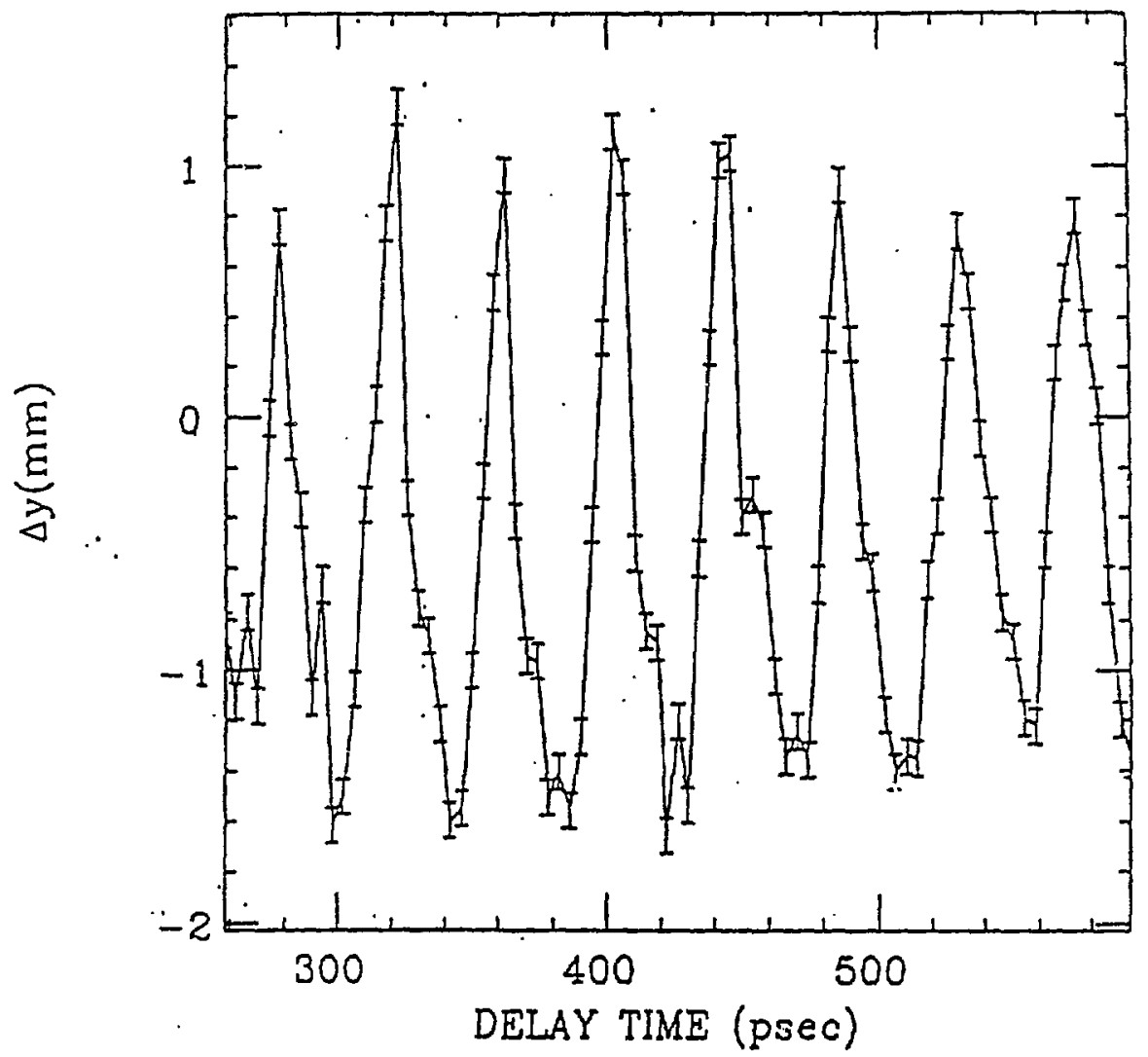

Figure 6 Deflecting wake field in a nonlinear plasma wave. 


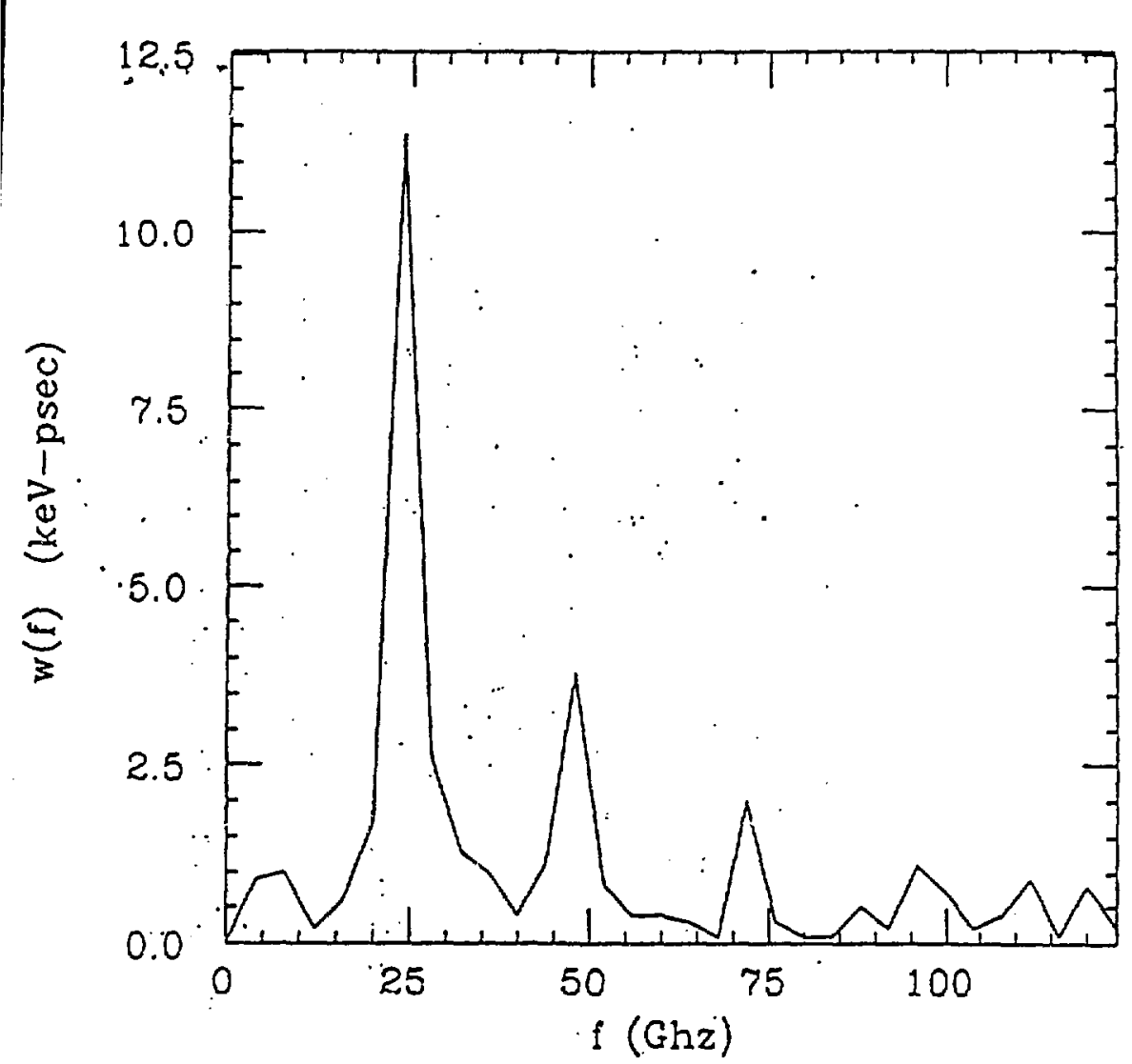

Tigure 7 (a) Fourier transform of longitudinal nonlinear plasma wake field.

Figure 7(b) Fourier transform of deflecting nonlinear plasma wake field.

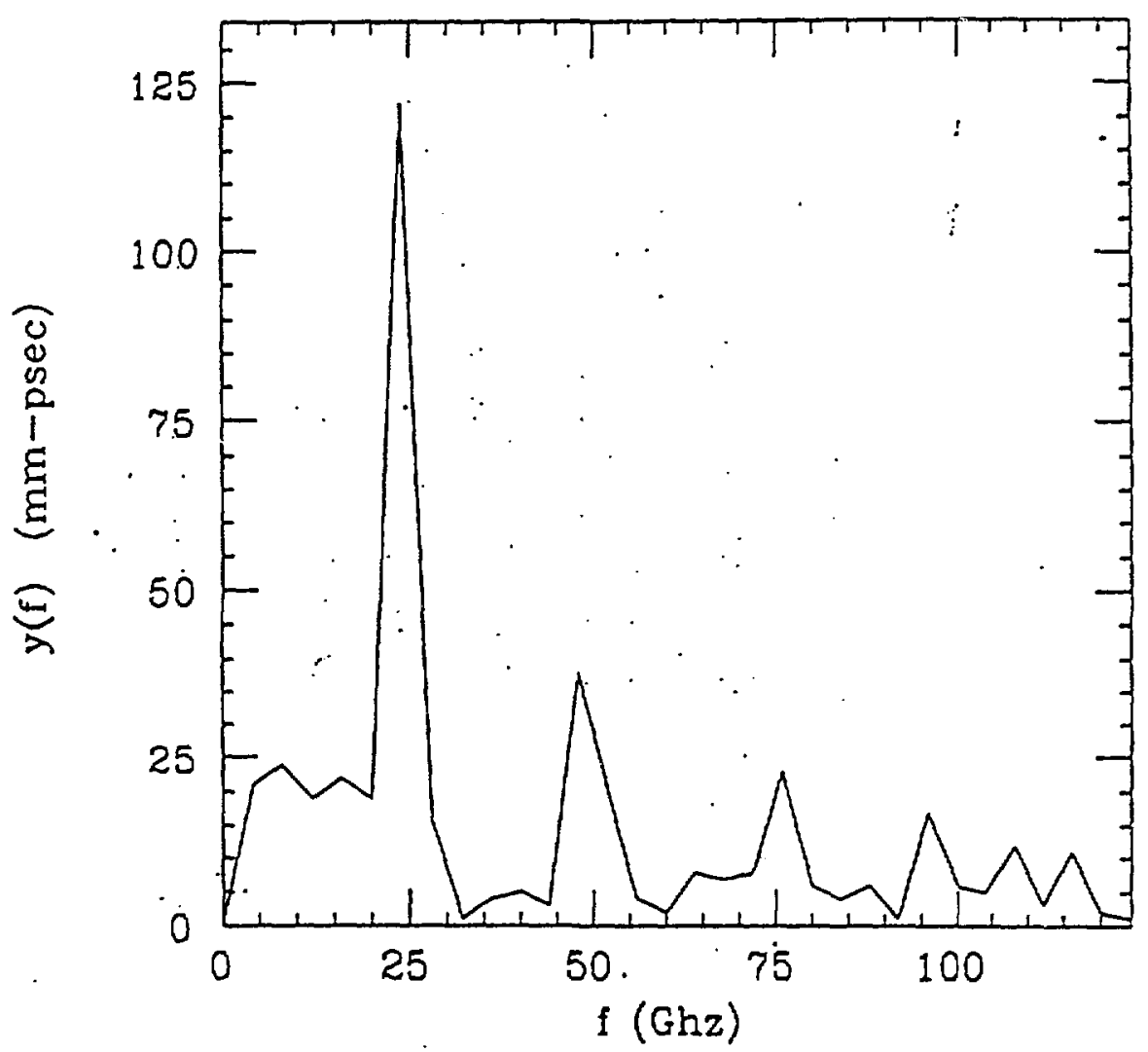

